\title{
Perfil Epidemiológico dos portadores de insuficiência renal crônica submetidos à terapia hemodialítica
}

Recebido em: 24/09/2013

Aprovado em: 12/08/2014
Ivonizete Pires Ribeiro' Andira Luana Soares Pinheiro² Andressa Luana Araújo Soares ${ }^{3}$ Nayara Franciele Marques dos Santos ${ }^{4}$

Resumo: Objetivou-se traçar o perfil epidemiológico do portador de IRC em tratamento hemodialítico na cidade de Teresina-PI. Tratou-se de um estudo quantitativo descritivo, realizado na unidade de nefrologia conveniada ao Sistema Único de Saúde (SUS), com 64 pacientes. A coleta de dados ocorreu de março a maio do ano de 2010 . Constatou-se que a maioria dos pacientes é do gênero masculino (67,19\%), faixa etária de 40 a 59 anos (40,63\%), sendo $60,94 \%$ casados, residentes em Teresina (64,06\%), com baixo nível de escolaridade, renda individual de 1 a 2 salários mínimos e com moradia própria. Em 69\% a doença de base foi hipertensão arterial e diabetes; e as principais dificuldades encontradas foram os efeitos causados pelo tratamento e o transporte, influenciando na manutenção da terapia hemodialítica. Tais fatores servem para subsidiar decisões para melhorar a assistência a esses pacientes juntamente com o enfermeiro atuando na prevenção das doenças que levam a IRC.

Descritores: Insuficiência Renal Crônica, Diálise Renal, Avaliação em enfermagem.

\section{Epidemiological profile of renal failure patients submitted to hemodialysis}

Abstract: The objective was to outline the epidemiological profile of patients with CRF on hemodialysis in the city of Teresina-PI. This is a descriptive quantitative study, conducted in the nephrology unit convening the National Health System (SUS), with 64 patients. Data collection occurred from March to May of 2010. It was found that the majority of patients are male (67.19\%), aged 40-59 years (40.63\%), and 60.94\% were married, living in Teresina $(64.06 \%)$ with fewer years of education, individual income 1-2 minimum wages and proper housing. In $69 \%$ the underlying disease was hypertension and diabetes, and the principal problems were the effects caused by treatment and transport, influencing the maintenance of hemodialysis. Such factors serve to support decisions to improve care for these patients jointly with nurses working in the prevention of diseases leading to CRF.

Descriptors: chronic renal failure, renal dialysis, nursing assessment.

\section{Perfil Epidemiológico dos los pacientes con insuficiencia renal crónica sometidos a hemodiálisis}

Resumen: El objetivo fue analizar el perfil epidemiológico de los pacientes con insuficiencia renal crónica en hemodiálisis en la ciudad de Teresina-PI. Este es un estudio cuantitativo descriptivo, realizado en la unidad de nefrología convocatoria del Sistema Nacional de Salud (SUS), con 64 pacientes. Los datos fueron recolectados entre marzo y mayo de 2010 . Se encontró que la mayoría de los pacientes son hombres (67,19\%), edad 40-59 años (40,63\%), y $60,94 \%$ estaban casadas, que viven en Teresina (64,06\%) con menos años de educación, los ingresos individuales de 1.2 salarios mínimos y una vivienda digna. En un 69\% la enfermedad de base fue la hipertensión y la diabetes, y los principales problemas fueron los efectos causados por el tratamiento y el transporte, que influyen en el mantenimiento de la hemodiálisis. Estos factores sirven para apoyar las decisiones para mejorar la atención de estos pacientes junto con las enfermeras que trabajan en la prevención de las enfermedades que conducen a la IRC.

Descriptores: Insuficiencia renal crónica, La diálisis renal, Evaluación en enfermería.

\section{INTRODUÇÃO}

A tualmente a IRC é um doença com alta taxa de morbimortalidade em todo o mundo. No Brasil, o censo da Sociedade Brasileira de Nefrologia (SBN) mostrou que, em março de 2008, havia 87.044 pacientes em diálise, um aumento de aproximadamente 21.923 pacientes em comparação a janeiro de 2005. Em Teresina-PI, a taxa de prevalência de pacientes em diálise é de 62,97 para cada 100 mil habitantes. Tendo como conseqüência o aumento da incidência desta doença crônica entre a população geral em âmbito local ${ }^{(1),(2)}$.

$\mathrm{O}$ aumento progressivo da incidência da IRC deve-se, em parte, ao incremento da prevalência de algumas enfermidades crônico-degenerativas destacando-se a hipertensão arterial sistêmica (HAS) e o diabetes mellitus (DM) (3).

A manutenção de pacientes em hemodiálise envolve custos elevados e é motivo de grande preocupação por parte de órgãos governamentais, que, no Brasil, subsidiam 95,0\% deste tratamento. Em 2002, foram gastos R\$ 1,4 bilhões no tratamento de pacientes em diálise crônica e com transplante renal em pacientes portadores de Insuficiência Renal Crônica - IRC(4).

Pessoas acometidas de doenças crônicas sofrem modificações no estilo de vida a que estavam acostumadas, como isolamento social, redução da atividade física, necessidade de adaptação, um sentimento ambíguo entre medo de viver e de morrer, e perda da autonomia relacionada ao tratamento constante podendo ocorrer no domicílio, nas clínicas e em alguns casos há ocorrência de internações. As reações do doente advêm do seu contexto social, cultural e das suas crenças e valores pessoais ${ }^{(5)}$.

Ao se identificar o perfil desta clientela atendida nas unidades de hemodiálise, o levantamento de dados poderá direcionar o enfermeiro e a equipe de saúde da família a adotar estratégias para um diagnóstico precoce das doenças crônico-degenerativas que são fatores de risco para a IRC.

A IRC define-se como uma deterioração progressiva e irreversível da função renal em que a capacidade do corpo para manter o equilíbrio metabólico e hidroeletrolítico falha, resultando em retenção de uréia e outros produtos de degradação nitrogenados do sangue ${ }^{(6)}$.

Esse estudo tem como objetivo traçar o perfil epidemiológico do portador de Insuficiência Renal Crônica (IRC) em tratamento hemodialítico na cidade de Teresina. 


\section{METODOLOGIA}

O estudo trata-se de uma investigação de natureza quantitativa e análise estatística descritiva, o campo selecionado para a realização da pesquisa foi o setor de hemodiálise da Clínica de Nefrologia do Hospital Getúlio Vargas. Foram entrevistados 64 pacientes portadores de insuficiência renal crônica, que concordaram em participar do estudo.

O material utilizado para o levantamento dos dados foram os prontuários dos pacientes e o instrumento utilizado para coletar os dados foram formulários estruturados com perguntas abertas e fechadas aplicados pelas autoras. Os dados foram coletados no período de março a maio de 2010 . Ao finalizarmos o levantamento dos dados, analisamos e organizando os dados numéricos em tabelas, utilizando como recurso o programa Statistical Product and Service Solutions - SPSS versão 16.0.

O projeto de pesquisa foi previamente enviado, analisado, e devidamente aprovado pelo Comitê de Ética em Pesquisa da Faculdade Novafapi em 15 de março de 2010, sob o Protocolo CAAE n. 0005.0.043.000-10. Além disso, todos os sujeitos que se disponibilizaram a participar do estudo, leram e assinaram o Termo de Consentimento Livre e Esclarecido, em acordo com a resolução 196/96 do Conselho Nacional de Saúde.

\section{RESULTADOS E DISCUSSÃO}

Tabela 1 - Perfil Sócio-demográfico da população estudada. Teresina(PI), 2010.

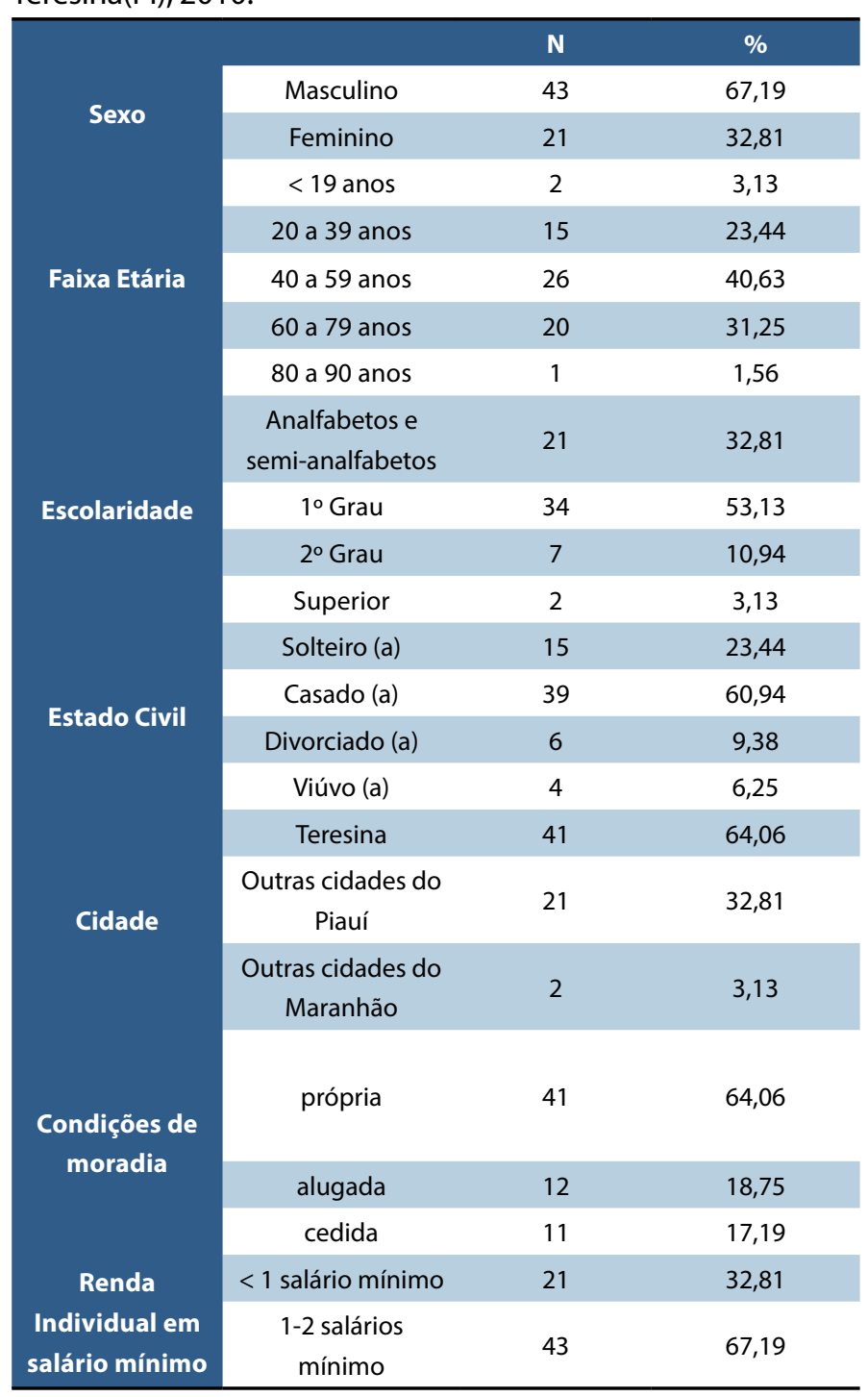

Quanto ao perfil demográfico (TAB. 1), constatou-se que $67,19 \%$ dos pacientes eram do sexo masculino. A distribuição da população por idade, apresentou predomínio de 40,63\% com faixa etária de 40 a 59 anos. Em relação ao estado civil 60,94\% eram casados, e 64,06\% residem em Teresina.

Quanto ao nível de instrução, dos 64 pacientes, 34 (53,13\%) possuíam apenas o ensino fundamental completo ou incompleto e 21 (32,81\%) eram analfabetos e semi-analfabetos. De acordo com os dados apresentados na TAB. 1, os pacientes com renda individual mensal entre 1 e 2 salários mínimos correspondem a maioria de $67,19 \%$, e os que residem em casa própria somam $64,06 \%$.

Neste estudo, a distribuição do acometimento da IRC, por gênero, mostrou maior freqüência de pessoas do sexo masculino, o que vem confirmar os dados obtidos de um estudo realizado no município de Anápolis-GO: entre 63 pacientes, 55,5\% eram do sexo masculino(7).

Os dados, encontrados no perfil da amostra estudada, sugerem que os homens procuram menos os serviços de saúde do que as mulheres, uma vez estas buscam os serviços de saúde devido a cuidados ginecológicos, o que as leva a se preocuparem mais com a saúde. Desta forma, os homens tornam-se mais suscetíveis à doença do que as mulheres.

Vale comentar que $40,63 \%$ correspondem à faixa etária dos pacientes analisados entre 40 a 59 anos. Este resultado representa um dado social significativo, pois a doença crônica atinge geralmente uma população em plena idade economicamente ativa, gerando gastos nas áreas sociais devido às aposentadorias precoces, gastos ambulatoriais e medicamentosos ${ }^{(8)}$.

Quanto ao estado civil, prevaleceu maior número de pessoas casadas $60,94 \%$ e os residentes na cidade de Teresina $64,06 \%$. Este perfil encontrado justifica-se pela maior prevalência de pessoas casadas, devido à cultura nesta região: nesta faixa etária intermediária, há um predomínio de pessoas casadas. A maior prevalência dos residentes na cidade de Teresina é devido à pesquisa ser realizada nesta cidade.

A escolaridade dos indivíduos pesquisados foi de $53,13 \%$ com o primeiro grau incompleto ou completo e taxa de $32,81 \%$ de analfabetos e semi-analfabetos. Desta forma, foi possível observar por meio de relatos dos pacientes, maior dificuldade para compreender as orientações preconizadas, repassadas pela equipe de saúde e a extensão do procedimento hemodialítico, o que pode comprometer ainda mais o estado de saúde do paciente em tratamento.

Os dados sobre a escolaridade dos participantes assemelham-se aos resultados obtidos em estudo desenvolvido em Curitiba, que buscou a relação do paciente renal crônico com o trabalho, evidenciando que, aproximadamente, $67,0 \%$ da amostra obtiveram apenas acesso ao ensino fundamental ${ }^{(9)}$.

De 64 pacientes, $67,19 \%$ apresentaram rendimentos compreendidos entre 1 e 2 salários mínimos, renda esta decorrente na maioria dos casos de benefícios da aposentadoria associada ao auxíliodoença. Destaca-se que $32,81 \%$ dos pacientes que possuem renda inferior a 1 salário mínimo, relatam não possuírem nenhum tipo de rendimento (donas-de-casa e desempregados). Não foram encontrados pacientes com renda superior a 2 salários mínimos.

Foram evidenciados fatores relacionadas ao processo de adoecimento em pacientes renais crônicos, demonstrando que $80,0 \%$ têm renda pessoal menor que 2 salários mínimos, o que caracteriza baixo nível sócio-econômico, pelo perfil de pacientes atendidos pelo SUS ${ }^{(10)}$. Estes resultados são similares 
aos encontrados na cidade de Teresina que também apresenta perfil de baixa renda individual.

Segundo esses dados, a população submetida à hemodiálise encontra-se inativa, do ponto de vista econômico, pois não possui fonte de renda, o que pode comprometer muito seu tratamento, já que a atividade laboral tem um papel fundamental na vida do homem ${ }^{(9)}$.

As condições de baixa renda são justificadas pela dificuldade de manter o emprego, devido ao tempo despendido na hemodiálise. Muitos pacientes utilizam, pelo menos, 6 horas do dia para tratamento, pois, após as 4 horas de hemodiálise, levam, aproximadamente, mais 2 horas para a recuperação dos sintomas imediatos após o tratamento, o que resulta na dificuldade do paciente manter um vínculo empregatício durante o seu tratamento(9).

Apesar do perfil sócio-demográfico ser considerado de baixa renda, $64,06 \%$ possuem moradia própria, casas simples com localização distanciada do centro da cidade devido aos preços populares que facilitam sua aquisição.

Tabela 2 - Hábito de vida por sexo. Teresina(PI) 2010.

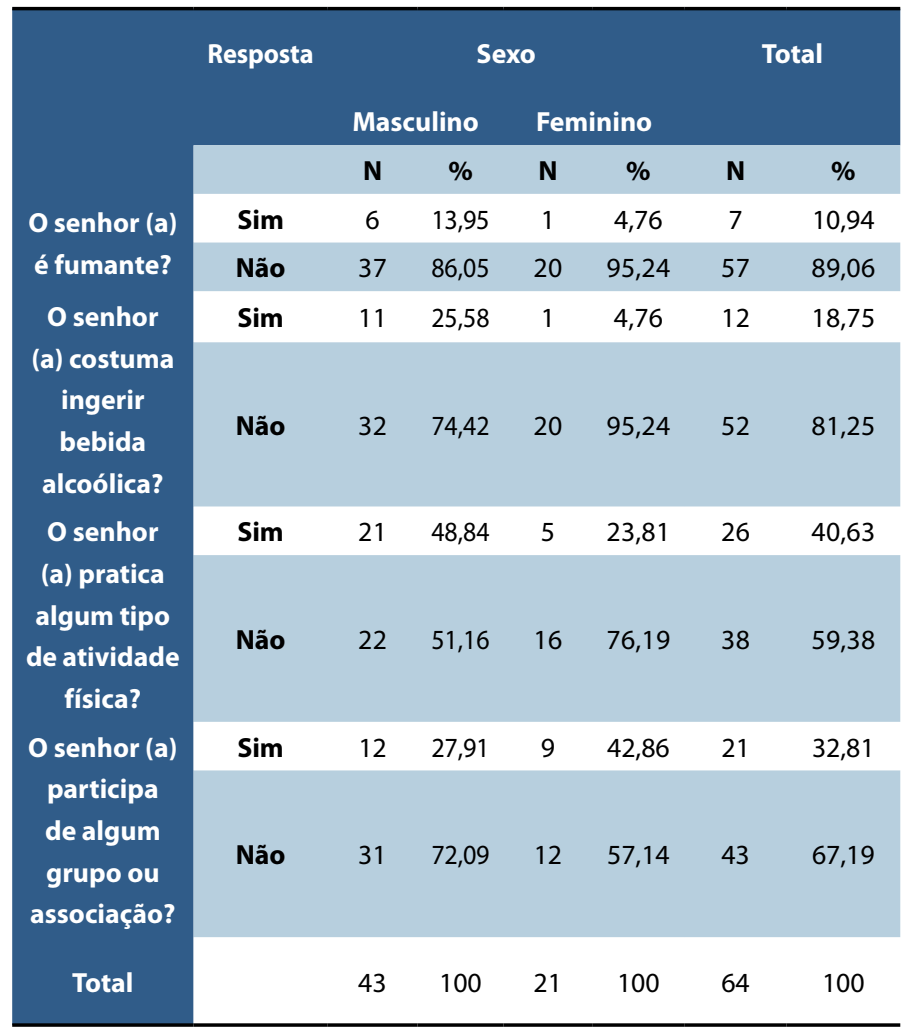

Em relação aos hábitos de vida dos pacientes (TAB. 2), constatou-se que a maioria tinha hábitos de vida sedentários $59,38 \%$ não pratica nenhum tipo de atividade física. Atualmente a causa do sedentarismo se dá devido aos efeitos causados pelo tratamento hemodialítico. Dos pacientes que praticam algum tipo de atividade física $(40,63 \%)$ após a IRC, a caminhada foi a mais relatada.

Quanto ao tabagismo, 10,94\% afirmam ser fumante, destes $13,95 \%$ são do sexo masculino. A maioria $89,06 \%$ nunca fumou ou abandonou o fumo em decorrência do tratamento da IRC. Neste estudo 81,25\% referiram não ingerir nenhum tipo de bebida alcoólica. Estudo realizado sugere que o tabagismo piora a sobrevida de pacientes em hemodiálise, pois diminui $72 \%$ em dois anos e $40 \%$ em seis anos nos fumantes ${ }^{(11)}$.

Dos pacientes entrevistados, $67,19 \%$ destes afirmam não participar de associações, grupos ou entidades, fato observado devido ao paciente depender da terapia hemodialítica para sobreviver, apresentando limitações no seu cotidiano após o início do tratamento sendo este responsável por um cotidiano monótono e restrito favorecendo o sedentarismo e a deficiência funcional, fatores que refletem diretamente na sua qualidade de vida ${ }^{(12)}$.

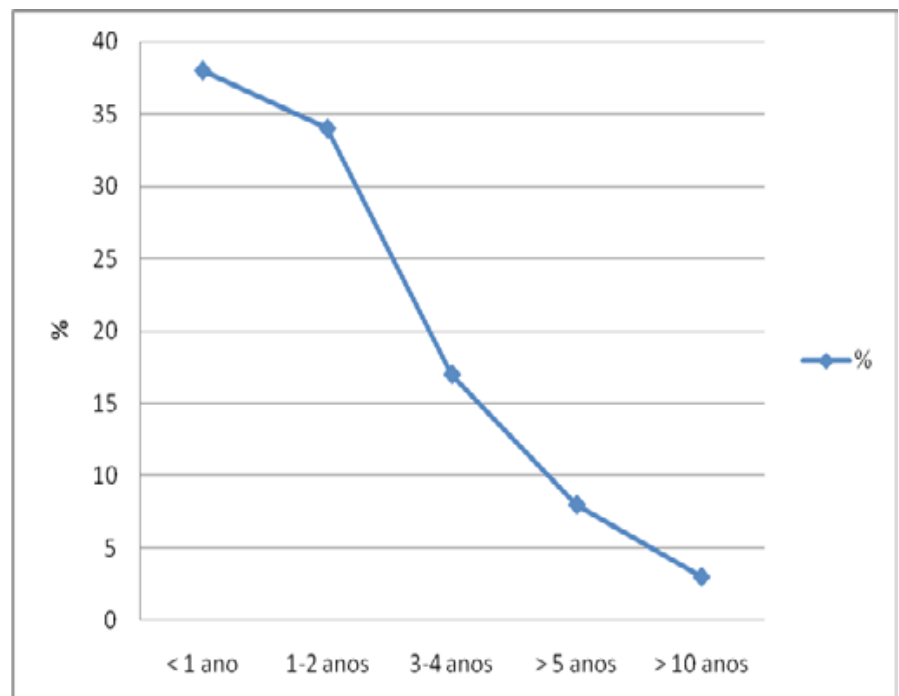

Gráfico 1 - Tempo de Tratamento Hemodialítico da população de estudo, Teresina-PI/2010.

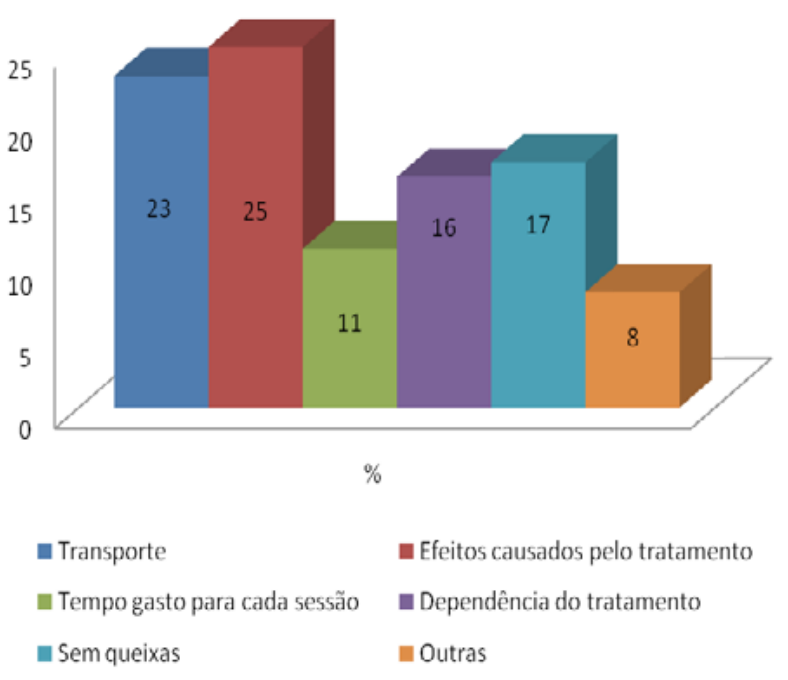

Gráfico 2 - Fatores que dificultam o tratamento da IRC no município deTeresina-PI/2010.

Entre os fatores mencionados como dificultadores do tratamento dos sujeitos do estudo (Gráfico 2), estão os efeitos causados pelo tratamento, que representou $25 \%$ das queixas, pelos pacientes apresentarem sintomas após a sessão como fraqueza, astenia e fadiga. Este fato está diretamente relacionado com uma grande parcela da população em estudo ter menos de 1 ano de tratamento hemodialítico, onde encontra-se os maiores efeitos devido a adaptação do organismo à terapia.

Em seguida os pacientes queixaram-se do transporte, sendo representado por $23 \%$. Mesmo havendo em Teresina a oferta da carteira de passe livre para todos os doentes renais crônicos 
em tratamento hemodialítico, e também a disponibilidade do transporte oferecido pela instituição onde é realizada a sessão de hemodiálise a locomoção se torna difícil. Isto deve-se ao fato da maior parte dos pacientes residirem na zona rural do município de Teresina, ficando fora da rota dos transportes públicos e do oferecido pela instituição.

O tempo gasto em cada sessão e sua periodicidade de 3 vezes por semana, são motivos de queixa de $11 \%$ dos pacientes, que relatam não ter tempo, nem disposição para executar atividades da vida diária antes realizadas. Já $8 \%$ dos pacientes relataram outras causas como dificuldades financeiras e a ausência de acompanhantes. A IRC produz alterações fisiológicas e funcionais, advindas da diminuição da atividade física, fraqueza muscular, anemia, alterações metabólicas, bem como acarreta prejuízos na qualidade de vida. Sendo assim, as queixas apresentadas mostram elementos que podem ser melhorados, a fim de gerar melhor qualidade de vida aos pacientes ${ }^{(14)}$.

Esses achados são significativos, porém, observou-se um contraste quando comparado a outro estudo em Anápolis-GO (2008), que mostra que entre as dificuldades encontradas a disponibilidade dos medicamentos (51\%) é a principal causa de queixa entre os pacientes, seguido de transporte $(20,5 \%)$ e tempo gasto para cada sessão (14,5\%), respectivamente. Aqueles que referiram dependência do tratamento e não ter queixas corresponde a $3 \%$ cada $^{(7)}$.

Para melhorar o tratamento e a qualidade de vida dos pacientes com IRC, faz-se necessária a simplificação do acesso ao transporte, que deve ser regulamentar, com a busca e o retorno dos pacientes no domicílio, uma vez que estes se sentem muito limitados, após as seções de hemodiálise. Com esta medida, é possível amenizar os custos que foram relatados por alguns entrevistados ${ }^{(7)}$.

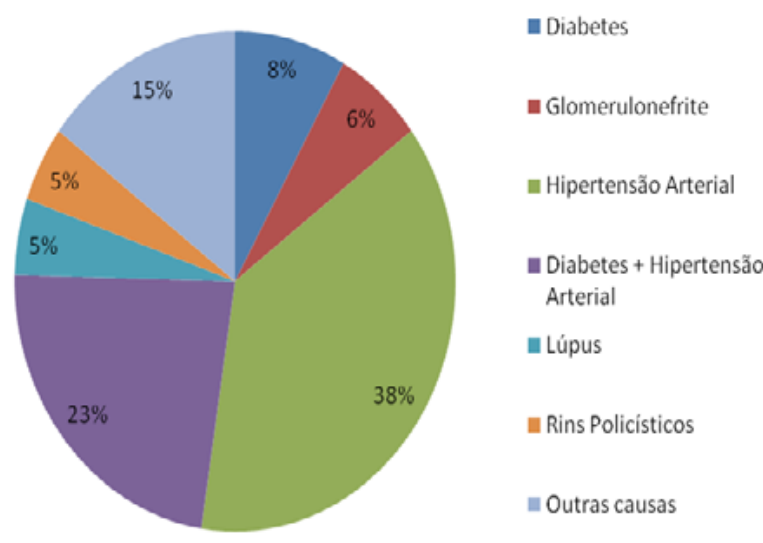

Gráfico 3 - Doenças de Base da insuficiência renal crônica, Teresina-PI/2010.

Foi constatado que as principais doenças de base para a IRC (Gráfico 3), são a hipertensão arterial (38\%), em seguida a associação de duas patologias - hipertensão e diabetes - que corresponde a $23 \%$, sendo que somente o diabetes corresponde a um total de $8 \%$. Além destas, outras moléstias estão relacionadas à perda da função renal, como a glomerulonefrite (6\%), rins policísticos e lúpus com $5 \%$ cada. No que diz respeito a outras causas que somam $15 \%$, destaca-se: cálculo renal, pielonefrite, abuso de medicamentos, agenesia renal, hipoplasia renal bilateral e acidentes que levaram a perca de um dos rins.

Os dados obtidos no estudo mostraram aproximação com resultados já obtidos em outras publicações, que apontam como principais causas de IRC a hipertensão arterial, diabetes mellitus, história familiar de IRC, idade avançada, glomerulopatias, doença renal policística, doenças auto-imunes, infecções sistêmicas, infecções urinárias de repetição, uropatias obstrutivas e neoplasias ${ }^{15}$.

A hipertensão arterial e o diabetes são responsáveis por cerca de metade das patologias dos pacientes em tratamento dialítico no Brasil. A hipertensão, relacionada ao diabetes, está intimamente ligada à reabsorção de água e sódio e à hiperatividade simpática mediadas pela insulina, bem como ao aumento na disponibilidade de cálcio intracelular. A insulina tem um efeito vasodilatador direto e a resistência a este efeito também contribui para a elevação da pressão arterial, trazendo uma sobrecarga aos rins, que leva à perda de suas funções ${ }^{(15),(16)}$.

Fatores predisponentes como estresse, alimentação inadequada, fumo, uso de hormônios estrogênicos, obesidade, sedentarismo e a falta de controle da saúde fazem com que o aumento da pressão arterial se torne inevitável ${ }^{(17)}$.

As estatísticas brasileiras são compatíveis àquelas de outros países da América Latina e menores do que as dos Estados Unidos, que evidenciam o diabetes e a hipertensão arterial como causas de IRC em três quartos dos pacientes em diálise. Esta diferença pode ser explicada pelo grande número de pacientes idosos em países desenvolvidos, uma vez que a expectativa de vida é maior, e também pelos hábitos alimentares e culturais destes países que favorecem o aparecimento destas doenças ${ }^{(15)}$.

\section{CONCLUSÃO}

Estes resultados epidemiológicos podem ser analisados como fatores interferentes na ocorrência da doença renal crônica, considerando as características sociais dos pacientes, tais como a baixa renda familiar e o baixo nível de escolaridade. Estes atributos podem influenciar no entendimento da importância do tratamento das doenças de base, do diagnóstico precoce e na prática do autocuidado.

O aumento em larga escala da incidência de patologias crônicas entre a população principalmente a hipertensão arterial, seguida da associação desta com diabetes, resulta na necessidade da atuação do enfermeiro na prevenção destas doenças por meio da estratégia de saúde da família (ESF), e pelas políticas públicas, para melhorar a qualidade de vida destes pacientes.

O convívio com uma doença crônica, em especial a insuficiência renal, reflete uma série de mudanças tanto na vida do paciente, como na de seus familiares, pois as limitações impostas pelo tratamento acarretarão diversas dificuldades, que interferem na sua qualidade de vida influenciando na manutenção do tratamento hemodialitico, e propiciando ao paciente algumas dificuldades de ordem sociais.

Os dados apresentados mostram diversos aspectos da população de pacientes com IRC em nosso meio, que servirão para subsidiar decisões para melhorar a assistência a esses pacientes, entre elas a criação e manutenção em longo prazo de um sistema nacional de registro, análise e divulgação de dados epidemiológicos de pacientes com IRC em tratamento renal substitutivo no país. 


\section{Referências}

\section{REFERÊNCIAS}

1. Sociedade brasileira de nefrologia (BR). Censo 2008. [on line] 2008; [citado 2009 set 05]. Disponível em: http://www.sbn.org.br/ Censo/2008/censo_SBN_2008.ppt

2. Ministério da saúde (BR). Sistema de informações ambulatoriais do SUS-SAI/SUS. [on line] 2006; [citado 2009 set 07]. Disponível em: http://tabnet.datasus.gov.br/cgi/tabcgi.exe?idb2007/d22.def.

3. Batista PBP, Lopes AA. Estudo Epidemiológico sobre a terapia renal substitutiva II. Epidemiol Serv Saúde. [on line] 2009 abr/jun; [citado 2009 set 13]; 18(2): [aprox.13 telas]. Disponível em: http:// www.scielo.br.

4. Sesso R. Epidemiologia da doença renal crônica no Brasil e sua prevenção. Secretaria de Estado da Saúde. Coordenadoria de Controle de Doenças. Centro de Vigilância Epidemiologia; São Paulo [periódico na internet]. 2006; [citado 2009 set 05]. Disponível em: ftp://ftp.cve.saude.sp.gov.br/doc_tec/cronicas/irc_prevprof. pdf.

5. Chaves LPD, Anselmi ML, Barbeira CBS, Hayashida M. Estudo da sobrevida de pacientes submetidos à hemodiálise e estimativa de gastos no município de Ribeirão Preto-SP. Rev Esc Enferm. [on line] 2002; [citado 2009 set 08]; 36(2): [aprox.10 telas]. Disponível em: http://www.scielo.br.

6. Smeltzer SC, Bare BG. Brunner e Suddarth: Tratado de enfermagem médico-cirúrgica. 6a ed. Rio de Janeiro: Guanabara Koogan; 2002.

7. Lima LR, Mendonça RR. Perfil epidemiológico do paciente renal crônico em tratamento hemodialítico em Anapólis - GO. Rev Intersec. [on line] 2008; [citado 2010 mai 23]; 9(2): [aprox.7 telas]. Disponível em: http://www.saocamilo-mg.br/publicacoes/ edicao_3/sao_camilo/artigo_04.pdf.

8. Barbosa DA, Gunji CK, Bittencourt ARC, Belasco AGS, Diccini S, Vattimo $F$, et al. Co-morbidade e mortalidade de pacientes em início de diálise. Acta paul Enferm. [on line] 2006; [citado 2010 mai 23]; 19(3): [aprox.6 telas]. Disponível em: http://www.scielo.br.
9. Lara EA, Sarquis LMM. O paciente renal crônico e sua relação com o trabalho. [CD-ROM] [Monografia]. Londrina: Curso de Enfermagem da Universidade Federal do Paraná, 2005.

10. Santos PR. Relação do Sexo e da Idade com Nível de Qualidade de Vida em Renais Crônicos Hemodialisados. Rev Assoc Méd Brasileira. [on line] 2006; [citado 2010 mai 23]; 52(5): [aprox.14 telas]. Disponível em: http://www.scielo.br.

11. Biernat JC. Sobrevida em hemodiálise. In: Cruz J, Barros RT, Sesso RCC, David Neto E, Suassuna JHR, Heilberg IP, et al, coordenadores. Atualidades em nefrologia 3. São Paulo: Savier; 1994. p. 155-159.

12. Martins MRI, Cesarino CB. Qualidade de vida de pessoas com doença renal crônica em tratamento hemodialítico. Rev Latino-am Enfermagem. [on line] 2005 set/out; [citado 2010 mai 24]; 13(5): [aprox.9 telas]. Disponível em: http://www.scielo.br.

13. Adjezen H, Schor N. Guia de nefrologia. 2ed. Barueri - SP Manole, 2005. p.468.

14. Pitta GBB, Andrade ART, Castro AA. Acesso venoso central para hemodiálise. Angiologia e cirurgia vascular: guia ilustrado UNCISAL/ECMAL \& LAVA [periódico na internet]. 2003; [citado 2010 maio 24]. Disponível em: http://www.lava.med.br/livro.

15. Associações de pacientes renais crônicos. Perfil da doença renal crônica: o desafio brasileiro 2007. [on line] 2007; [citado 2010 mai 24]. Disponível em: http://www.sbn.org.br/noticias/DossieFinal. pdf.

16. Romão Jr JE. O rim e suas doenças. Sociedade Brasileira de Nefrologia. [on line] 2004. [citado 2010 mai 24]. Disponível em: http://www.sbn.org.br/Publico/rim.htm.

17. Pinheiro ME, Alves CMP. Hipertensão arterial na diálise e no transplante renal. J Bras Nefrol. [periódico na internet]. 2003; [citado 2010 maio 24]; 25(3): [aprox.13 telas]. Disponível em: http:// www.sbn.org.br/JBN/JBNAcontece/0203/revisao1.htm. 